\title{
A Quasi-3-D Theory for Impedance Eduction in Uniform Grazing Flow
}

\author{
W. R. Watson, M. G. Jones, and T. L. Parrott ${ }^{\ddagger}$ \\ NASA Langley Research Center, Hampton, Virginia 23681-2199, USA
}

\begin{abstract}
A 2-D impedance eduction methodology is extended to quasi-3-D sound fields in uniform or shearing mean flow. We introduce a nonlocal, nonreflecting boundary condition to terminate the duct and then educe the impedance by minimizing an objective function. The introduction of a parallel, sparse, equation solver significantly reduces the wall clock time for educing the impedance when compared to that of the sequential band solver used in the 2-D methodology. The accuracy, efficiency, and robustness of the methodology is demonstrated using two examples. In the first example, we show that the method reproduces the known impedance of a ceramic tubular test liner. In the second example, we illustrate that the approach educes the impedance of a four-segment liner where the first, second, and fourth segments consist of a perforated face sheet bonded to honeycomb, and the third segment is a cut from the ceramic tubular test liner. The ability of the method to educe the impedances of multisegmented liners has the potential to significantly reduce the amount of time and cost required to determine the impedance of several uniform liners by allowing them to be placed in series in the test section and to educe the impedance of each segment using a single numerical experiment. Finally, we probe the objective function in great detail and show that it contains a single minimum. Thus, our objective function is ideal for use with local, inexpensive, gradient-based optimizers.
\end{abstract}

\section{Nomenclature}

$A_{m n} \quad=$ mode coefficients

$\{B\} \quad=4 \mathrm{MN} \times 1$ vector of source effects

$c_{0}, \rho_{0} \quad=$ ambient sound speed and density

$[C],[G]=M \times M$ matrices defining the nonlocal boundary condition

$\{D\},\{P\}=M \times 1$ column vectors containing mode amplitudes and mode pressures

$\{U\} \quad=M \times 1$ column vector containing the axial derivative of mode pressures

$D / D t \quad=\partial / \partial t+\vec{U}_{0} \bullet \vec{\nabla}$, material derivative

$E(k, \ldots)=$ transcendental function for determining eigenvalues

$f \quad=$ source frequency

$F(\zeta) \quad=$ objective function for impedance eduction

$g_{m}(x) \quad=$ normal derivative of $3-\mathrm{D}$ source function

$G_{m}(x)=3$-D source functions

$H, L, W=$ height, length, and width of the duct

$i=\sqrt{-1}$, unit complex number

$k=2 \pi f / c_{0}$, freespace wavenumber

$K_{m n} \quad=$ axial propagation constant

$L_{1}, L_{2} \quad=$ leading and trailing edge of test liner

$M, N \quad=$ number of evenly spaced vertical and axial points

$M_{0} \quad=$ uniform flow Mach number

$M_{\text {ave }} \quad$ = average mean flow Mach number in Grazing Incidence Tube

$\tilde{p}, \tilde{\rho} \quad=3-\mathrm{D}$ time dependent acoustic pressure and density

*Senior Research Scientist, Research and Technology Directorate, Computational AeroSciences Branch, Mail Stop 128, Willie.R.Watson@NASA.Gov. Senior Member AIAA.

${ }^{\dagger}$ Research Scientist, Research and Technology Directorate, Structural Acoustics Branch, Mail Stop 463, Michael.G.Jones@ NASA.Gov. Senior Member AIAA.

${ }^{\ddagger}$ Senior Research Scientist, Research and Technology Directorate, Structural Acoustics Branch, Mail Stop 463, Tony.L.Parrott@ NASA.Gov. 
$p, P_{m} \quad=3-\mathrm{D}$ and quasi-3-D time-harmonic acoustic pressure field

$p_{s}, p_{n} \quad=$ source plane pressure and axial derivative of source plane pressure

$Q_{m n}(x)=$ mode eigenfunction in the vertical direction

$\theta, \xi=$ normalized resistance and reactance

$[S] \quad=4 \mathrm{NM} \times 4 \mathrm{NM}$ stiffness matrix

$t \quad=$ time

$\tilde{u}, \tilde{v}, \tilde{w}=$ vertical, spanwise, and axial acoustic particle velocity components

$\overrightarrow{\vec{u}}, \vec{U}_{0} \quad=$ acoustic particle velocity and mean velocity vector

$w_{0} \quad=$ axial component of mean velocity

$x, y, z=$ Cartesian coordinates

$[Z] \quad=$ dimensionless node impedance matrix

$\alpha_{m n} \quad=$ transcendental function coefficient

$\nabla^{2}, \vec{\nabla} \quad=3$-D Laplace operator and gradient operator

$\zeta, \zeta_{\text {exit }}=$ test liner and exit plane impedance

$\zeta_{l}, \zeta_{u} \quad=$ impedance of lower and upper wall downstream of the test liner

$\omega=2 \pi f$, angular frequency

$\lambda_{n} \quad=$ mode eigenvalue in the vertical direction

$\{\Phi\} \quad=4 M N \times 1$ vector of nodal acoustic pressures

$\partial / \partial n=$ the derivative normal to the liner surface

- $\quad=$ dot product

Superscripts:

$-1=$ matrix inverse

* $\quad=$ complex conjugate

FEM = finite element computed wall pressure

Meas $=$ measured wall pressure

Subscripts:

$m, n \quad=$ spanwise and vertical mode order

$0=$ mean flow quantity

$J \quad=$ wall location index

\section{Introduction}

$E^{n}$ FFICIENT duct liners for broadband acoustic noise suppression are critical to the development of environmentally acceptable commercial aircraft. Previous experiments have shown that significant changes in duct liner impedance can occur as a result of grazing flow. ${ }^{1}$ Because of the need to better understand this phenomenon, NASA Langley Research Center (LaRC) has invested a considerable effort into the development of finite element models for educing liner impedance in the presence of grazing flow. In this approach, acoustic data needed for implementation of the finite element methodology is acquired in the test section of the $51 \mathrm{~mm} \times 51 \mathrm{~mm}$ Grazing Incidence Tube (GIT). This methodology seeks to completely describe the aeroacoustic field interior to and on the boundaries of a computational domain centered on the test liner (see Fig. 1). Impedances educed using this method have been extensively reported, and the methodology has steadily evolved from the no-flow standard of several years ago ${ }^{2}$ to a uniform flow ${ }^{3}$, and now, to a sheared flow model. ${ }^{4}$ To date, this methodology has been limited to 2-D aeroacoustic sound fields.

Recently, results from the finite element method were compared to results of methods used in various U.S. aeroacoustics laboratories. ${ }^{5}$ Impedance data comparisons acquired from this multilaboratory study demonstrated the need to incorporate 3-D aeroacoustic effects into the impedance eduction methodologies. Subsequently, a 95-microphone test window was installed in the GIT $^{6}$ to obtain data for impedance eduction in 3-D aeroacoustics sound fields. Additionally, LaRC is building a large-scale flow duct (LSFD) that is closer in size to a full-scale engine. Because the cross-sectional area of the LSFD is more than 22 times that of the GIT, transverse modes (and hence 3-D acoustic effects) occur over the desired range of test frequencies ( 0.5 to $3.0 \mathrm{kHz})$. Thus, an ability to educe liner impedance in 3-D aeroacoustic sound fields is clearly needed.

This paper describes modifications to the LaRC 2-D finite element methodology that facilitate studies of impedance eduction in 3-D aeroacoustic sound fields with grazing flow. Although the method presented in this paper is applicable 
to shearing mean flows, the results presented herein have been restricted to uniform mean flow in order to limit the scope of the current investigation. The paper reports initial results achieved in the GIT with these modifications using data acquired with a ceramic tubular test liner installed. The methodology is then applied in a geometry corresponding to the LSFD to demonstrate the portability of the technology to other geometries and to 3-D aeroacoustic sound fields. The paper also illustrates how to use the methodology to educe impedances of multisegmented liners in a flow.

\section{Statement of Problem}

$\mathrm{F}$ GURE 1 shows a schematic of the 3-D duct and right-hand Cartesian coordinate system used in this study, together with the computational domain. The source and exit planes are located at $z=0$ and $z=L$, respectively, as illustrated in the figure. The test liner is installed in the lower wall with its leading and trailing edge located at $z=L_{1}$ and $z=L_{2}$, respectively. The portions of duct upstream and downstream of the leading and trailing edges are considered rigid. The test liner is assumed locally reacting (i.e., wave motion in the liner is restricted to the $\mathrm{x}$-direction, as shown in Fig. 1) and has an unknown normalized time-harmonic impedance, $\zeta$, that is generally a function of wall location. Although the test liner is shown on the lower wall in Fig. 1, the analysis to follow will allow the liner to be placed on either the upper or lower wall of the duct. Throughout this work all wall impedances are normalized with respect to the characteristic impedance $\left(\rho_{0} c_{0}\right)$ of the air flowing in the duct and all dimensions are in mks units. It is further assumed that the time-harmonic acoustic pressure field on the wall opposite the test liner is known. (These could be provided, for example, by a 95-microphone window such as that discussed in Ref. 6). Finally, there is a steady flow velocity vector, $\vec{U}_{0}$, within the duct that flows from left to right at a subsonic speed. The problem at hand is to determine the unknown impedance, $\zeta$, of the test liner in the presence of the viscous, compressible, steady flow field.

\section{Governing Differential Equations}

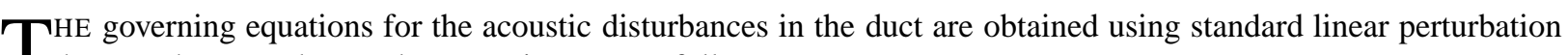
theory. The procedure and assumptions are as follows:

1. The fluid in the duct is assumed to be an ideal gas.

2. The steady flow is obtained from the steady solution of the Navier Stokes equations or from measurements.

3. The acoustic equations are derived assuming that each time-dependent flow quantity may be represented as the sum of the known steady flow and a time dependent (acoustic) component. The sum of these two fields is assumed to collectively satisfy the unsteady Navier Stokes equations (turbulence is included statistically in the mean flow).

4. The nonlinear acoustic equations are then obtained by subtracting off the steady flow equations from the timedependent equations.

5. We further simplify the acoustic equations by assuming the acoustic process is isentropic. Thus, viscous and heat conduction effects are assumed important only in establishing the steady flow field, setting up the sources of sound, and dissipating initial transients.

6. The resulting nonlinear acoustic equations are then linearized about the steady flow to obtain the governing equations for linear acoustic disturbances in the flowing fluid.

When written in compact vector format in Cartesian coordinates $(x, y, z)$, the linearized acoustic equations are ${ }^{7}$

$$
\begin{gathered}
\frac{D \tilde{\rho}}{D t}+\rho_{0}(\vec{\nabla} \bullet \overrightarrow{\tilde{u}})+(\overrightarrow{\tilde{u}} \bullet \vec{\nabla}) \rho_{0}=0 \\
\frac{D}{D t}=\frac{\partial}{\partial t}+\vec{\nabla} \bullet \vec{U}_{0} \\
\rho_{0}\left[\frac{D \overrightarrow{\vec{u}}}{D t}+(\overrightarrow{\vec{u}} \bullet \vec{\nabla}) \vec{U}_{0}\right]=-\vec{\nabla} \tilde{p} \\
\tilde{p}=c_{0}^{2} \tilde{\rho}
\end{gathered}
$$


Equations (1)-(4) constitutes a system of five equations in five unknown acoustic variables $(\tilde{\rho}, \tilde{p}, \tilde{u}, \tilde{v}, \tilde{w})$. These equations along with appropriate initial conditions, wall boundary condition, exit plane condition, and inflow boundary conditions, constitute a well-posed initial boundary value problem that can be evolved to a quasi-periodic state. Given the time-harmonic impedance, $\zeta$, this problem can be solved to uniquely determine the time-harmonic acoustic pressure on the wall opposite the test liner. Conversely, if the time-harmonic acoustic pressure on the wall opposite the test liner is known (e.g., measured), the unknown impedance may be iteratively determined. The procedure would be to cycle through the solution in the duct for each impedance. The impedance that reproduces the known acoustic pressure opposite the test liner is the impedance of the test liner. The authors have referred to this procedure as impedance eduction in the duct acoustics literature. ${ }^{3-6}$

Henceforth, we assume that the mean flow in the duct is a fully developed parallel flow. This assumption focuses attention far downstream of the duct entrance, so that the initial growing shear layer has vanished, and the steady flow velocity vector $\vec{U}_{0}$ is purely axial and varies only with the two transverse coordinates; that is $\vec{U}_{0}=\left(0,0, w_{0}(x, y)\right)$. The assumption of a region of parallel steady flow at some finite distance downstream of the duct entrance is a reasonable approximation in many laboratory situations.

The assumption of a parallel steady flow field allows a decoupling of the axial component of acoustic particle velocity $(\tilde{w})$ from the continuity of mass flow and the two transverse momentum equations:

$$
\begin{aligned}
\frac{1}{c_{0}^{2}} \frac{D^{2} \tilde{p}}{D t^{2}}-\nabla^{2} \tilde{p}-2 \rho_{0} c_{0}\left[\frac{\partial M_{0}}{\partial x} \frac{\partial \tilde{u}}{\partial z}+\frac{\partial M_{0}}{\partial y} \frac{\partial \tilde{v}}{\partial z}\right]=0 \\
\frac{D \tilde{u}}{D t}=-\frac{1}{\rho_{0}} \frac{\partial \tilde{p}}{\partial x} \\
\frac{D \tilde{v}}{D t}=-\frac{1}{\rho_{0}} \frac{\partial \tilde{p}}{\partial y} \\
M_{0}=\frac{w_{0}}{c_{0}}
\end{aligned}
$$

The above system (Eqs. (5)-(7)) constitutes three equations in the three unknown variables. $\tilde{p}, \tilde{u}$, and $\tilde{v}$, that can be solved to obtain the acoustic disturbances in the flow. It is convenient to combine these equations into a single equation involving the acoustic pressure field. The motivation for considering only an equation on acoustic pressure is that a pressure equation requires knowledge of only the boundary pressure disturbances to generate the appropriate boundary conditions. On the other hand, a solution to the above system (Eqs. (5)-(7)) will ultimately require that some components of the acoustic particle velocity vector be known at inflow to obtain the appropriate number of boundary conditions. Because the inflow boundary conditions are typically measured, and the technology for measuring acoustic pressure disturbances is better developed than that for measuring acoustic particle velocity, this paper focuses on the solution to only a single pressure equation.

To arrive at a single equation involving the acoustic pressure field, the transverse components of acoustic particle velocities are eliminated from Eqs. (5)-(7). This gives a single but third order partial differential equation that governs the acoustic pressure disturbance in the duct ${ }^{7}$

$$
\frac{1}{c_{0}^{2}} \frac{D^{3} \tilde{p}}{D t^{3}}-\frac{D}{D t}\left(\nabla^{2} \tilde{p}\right)+2 c_{0}\left[\frac{\partial M_{0}}{\partial x} \frac{\partial^{2} \tilde{p}}{\partial z \partial x}+\frac{\partial M_{0}}{\partial y} \frac{\partial^{2} \tilde{p}}{\partial z \partial y}\right]=0
$$

Here we are interested in only time harmonic solutions for the acoustic pressure field. These are of the form

$$
\tilde{p}=p e^{i \omega t}
$$

Thus, for uniform flow under time harmonic conditions, Eq. (5) reduces to the convected Helmholtz equation

$$
\left(1-M_{0}^{2}\right) \frac{\partial^{2} p}{\partial z^{2}}+\frac{\partial^{2} p}{\partial x^{2}}+\frac{\partial^{2} p}{\partial y^{2}}-2 i k M_{0} \frac{\partial p}{\partial z}+k^{2} p=0
$$

and the time harmonic form of Eq. (9) for parallel shearing mean flow is

$$
\begin{gathered}
M_{0}^{3} \frac{\partial^{3} p}{\partial z^{3}}-M_{0} \frac{\partial}{\partial z} \nabla^{2} p+3 i k M_{0}^{2} \frac{\partial^{2} p}{\partial z^{2}}-i k \nabla^{2} p+ \\
2\left(\frac{\partial M_{0}}{\partial x} \frac{\partial^{2} p}{\partial z \partial x}+\frac{\partial M_{0}}{\partial y} \frac{\partial^{2} p}{\partial z \partial y}\right)-3 k^{2} M_{0} \frac{\partial p}{\partial z}-i k^{3} p=0
\end{gathered}
$$


It remains to be demonstrated in a future study as to whether this derivation is sufficiently computationally robust and can be implemented using a practical number of acoustic pressure measurement locations. For now, it is exciting to note that this approach offers potential for usage in impedance eduction with shorter liners (e.g., $0.051 \mathrm{~m} \mathrm{long}$ ) in the presence of much higher mean flow velocities, which can be achieved using converging-diverging inserts in the GIT. Over this shorter length, it may be acceptable to assume that the mean flow is parallel sheared flow. If successful, this would significantly enhance our impedance eduction capability.

\section{Acoustic Boundary Conditions}

$\mathrm{T}_{\mathrm{c}}^{\mathrm{o}}$ obtain a unique solution to the acoustic equations (Eqs. (11) or (12)) and educe the impedance, boundary conditions must be prescribed. These acoustic equations require a wall boundary condition at the duct walls, a source boundary condition at the source plane $(z=0)$, and an exit boundary condition at the exit plane of the duct $(z=L)$. In addition, a normal mode analysis shows that the third order equation requires a second inflow boundary condition. The locally reacting, impedance boundary condition for an impenetrable surface without curvature and arbitrary steady flow has been presented by Myers ${ }^{8}$

$$
\frac{\partial p}{\partial n}=i k\left[\frac{p}{\zeta}\right]+2 M_{0} \frac{\partial}{\partial z}\left[\frac{p}{\zeta}\right]+\frac{M_{0}^{2}}{i k} \frac{\partial^{2}}{\partial z^{2}}\left[\frac{p}{\zeta}\right]
$$

where the acoustic impedance is set to infinity $(\zeta=\infty)$ along a rigid wall portion of the duct. At the source plane the acoustic pressure is assumed known

$$
p=p_{s}
$$

In addition, in a parallel shearing mean flow, we will specify the derivative of acoustic pressure normal to the inflow boundary as the second inflow boundary condition

$$
\frac{\partial p}{\partial z}=p_{n}
$$

The derivative of the acoustic pressure normal to the inflow boundary $\left(p_{n}\right)$ can be obtained using the 95-microphone window that was discussed in a previous paper. ${ }^{6}$ At the duct termination a variety of boundary conditions that render the boundary value problem on the acoustic pressure field well-posed could be implemented. When only the plane wave mode is cut on at the exit plane, it is convenient to specify an exit impedance condition, so that the boundary condition will be of the form

$$
\frac{\partial p}{\partial z}=-\frac{i k p}{\left(M_{0}+\zeta_{\text {exit }}\right)}
$$

The above exit impedance boundary condition was used in our previous 2-D analysis. ${ }^{3-5}$ Note that the exit impedance boundary condition (Eq. (16)) is a boundary condition with constant coefficients that is easily implemented into our impedance eduction procedure. However, when higher-order modes are present at the termination, the constant coefficient exit impedance boundary condition is not appropriate.

An alternative to the exit impedance boundary condition in the presence of higher-order modes is the nonreflecting boundary condition. Physically, implementing a nonreflecting boundary condition at the exit plane is equivalent to attaching an infinite length of duct downstream of the exit boundary so that downstream propagating waves are not reflected. Such a condition is generally accepted as being physically correct when the portion of duct downstream of the trailing edge of the test liner is extremely long. In this work we apply a generalization of a zero-flow, nonreflecting boundary condition presented in an earlier work. ${ }^{9}$ A detailed derivation of the nonreflecting exit plane boundary condition that includes mean flow effects is presented in a subsequent section.

\section{Quasi-3-D Analysis}

$\mathrm{T}^{\mathrm{N}}$ this paper it is convenient to develop the impedance eduction theory in Quasi-3-D. The quasi-3-D theory greatly Ireduces the computational time and memory required to educe the impedance by reducing the 3-D problem to a series of 2-D calculations. However, the quasi-3-D model still adequately represents many of the essential features of a fully 3-D theory. The quasi-3-D theory is applicable when the solution in the spanwise direction is separable (e.g., when one or both sidewalls of a duct are lined with uniform acoustic treatment and the mean flow is independent of the spanwise coordinate). When the sidewalls of the duct are rigid as in Fig. 1, the acoustic pressure field and sound 
source may be expanded into the following Fourier series of hard wall duct modes

$$
\begin{aligned}
p(z, x, y) & =\sum_{m=0}^{m=\infty} P_{m}(z, x) \cos \left(\frac{m \pi y}{W}\right) \\
p_{s}(x, y) & =\sum_{m=0}^{m=\infty} G_{m}(x) \cos \left(\frac{m \pi y}{W}\right) \\
p_{n}(x, y) & =\sum_{m=0}^{m=\infty} g_{m}(x) \cos \left(\frac{m \pi y}{W}\right)
\end{aligned}
$$

where $m$ takes on integer values, and the functions $G_{m}(x)$ and $g_{m}(x)$ are known from the source condition.

Thus, the partial differential equation, wall, and source plane boundary conditions that govern impedance eduction in uniform or parallel shearing flow are now summarized. In a uniform flow the differential equation is

$$
\left(1-M_{0}^{2}\right) \frac{\partial^{2} P_{m}}{\partial z^{2}}+\frac{\partial^{2} P_{m}}{\partial x^{2}}-2 i k M_{0} \frac{\partial P_{m}}{\partial z}+\left[k^{2}-\left(\frac{m \pi}{W}\right)^{2}\right] P_{m}=0
$$

the wall boundary condition becomes

$$
\frac{\partial P_{m}}{\partial n}=i k\left[\frac{P_{m}}{\zeta}\right]+2 M_{0} \frac{\partial}{\partial z}\left[\frac{P_{m}}{\zeta}\right]+\frac{M_{0}^{2}}{i k} \frac{\partial^{2}}{\partial z^{2}}\left[\frac{P_{m}}{\zeta}\right]
$$

and inflow boundary condition is

$$
P_{m}=G_{m}(x)
$$

On the other hand, when there is shear flow, the differential equation is

$$
\begin{gathered}
\left(M_{0}^{3}-M_{0}\right) \frac{\partial^{3} P_{m}}{\partial z^{3}}-M_{0} \frac{\partial^{3} P_{m}}{\partial z \partial x^{2}}-i k \frac{\partial^{2} P_{m}}{\partial x^{2}}-i k\left(1-3 M_{0}^{2}\right) \frac{\partial^{2} P_{m}}{\partial z^{2}}+ \\
2 \frac{d M_{0}}{d x} \frac{\partial^{2} P_{m}}{\partial x \partial z}+\left[M_{0}\left(\frac{m \pi}{W}\right)^{2}-3 k^{2} M_{0}\right] \frac{\partial P_{m}}{\partial z}-i k\left[k^{2}-\left(\frac{m \pi}{W}\right)^{2}\right] P_{m}=0
\end{gathered}
$$

the wall boundary condition becomes

$$
\frac{\partial P_{m}}{\partial n}=i k\left[\frac{P_{m}}{\zeta}\right]+2 M_{0} \frac{\partial}{\partial z}\left[\frac{P_{m}}{\zeta}\right]+\frac{M_{0}^{2}}{i k} \frac{\partial^{2}}{\partial z^{2}}\left[\frac{P_{m}}{\zeta}\right]
$$

and the inflow boundary conditions become

$$
\begin{gathered}
P_{m}=G_{m}(x) \\
\frac{\partial P_{m}}{\partial z}=g_{m}(x)
\end{gathered}
$$

Equations (20)-(22) for uniform flow or equations (23)-(26) for parallel shearing flow, along with the appropriate nonreflecting, exit plane boundary condition, constitute a well-posed boundary value problem for the unknown pressure function $P_{m}(z, x)$. Exact analytical solutions for $P_{m}(z, x)$ are available for infinitely long ducts. This infinite duct analytical solution is derived in the following subsection and its derivation is essential for formulating the proper mathematical form of the nonreflecting boundary condition at the duct exit.

\section{Nonreflecting Boundary Condition}

Nonreflecting boundary condition at the exit boundary is now developed. This boundary condition can be used A provided that the duct termination is nonreflecting. The nonreflecting boundary condition developed here is a generalization of the zero flow boundary condition developed in an earlier work. ${ }^{9}$ The development begins by expanding $P_{m}$ and its axial derivative at the exit plane as a series of right-moving duct modes

$$
P_{m}=\sum_{n=1}^{M} A_{m n} Q_{m n}(x) e^{-i K_{m n} z}
$$




$$
\frac{\partial P_{m}}{\partial z}=-i \sum_{n=1}^{M} K_{m n} A_{m n} Q_{m n}(x) e^{-i K_{m n} z}
$$

We now divide the vertical coordinate, $x$, into $M$ evenly spaced nodes, located at $x=x_{1}, x=x_{2}, \ldots x=x_{M}$, across the exit of the duct. Evaluating the series in Eqs. (27) and (28) at each boundary node point gives

$$
\{P\}=[C]\{D\}
$$

and

$$
\begin{gathered}
\{U\}=-i k[C][G]\{D\} \\
\{P\}=\left\{\begin{array}{c}
P_{m}\left(L, x_{1}\right) \\
P_{m}\left(L, x_{2}\right) \\
P_{m}\left(L, x_{3}\right) \\
\vdots \\
P_{m}\left(L, x_{M}\right)
\end{array}\right\},[C]=\left[\begin{array}{cccc}
Q_{m, 1}\left(x_{1}\right) & Q_{m, 2}\left(x_{1}\right) & \cdots & Q_{m, M}\left(x_{1}\right) \\
Q_{m, 1}\left(x_{2}\right) & Q_{m, 2}\left(x_{2}\right) & \cdots & Q_{m, M}\left(x_{2}\right) \\
\vdots & \vdots & \ddots & \vdots \\
Q_{m, 1}\left(x_{M}\right) & Q_{m, 2}\left(x_{M}\right) & \cdots & Q_{m, M}\left(x_{M}\right)
\end{array}\right] \\
\{D\}=\left\{\begin{array}{c}
A_{m, 1} e^{-i K_{m, 1} L} \\
A_{m, 2} e^{-i K_{m, 2} L} \\
A_{m, 3} e^{-i K_{m, 3} L} \\
\vdots \\
A_{m, M} e^{-i K_{m, M} L}
\end{array}\right\} \\
\{U\}=\frac{\partial}{\partial z}\left\{\begin{array}{c}
P_{m}\left(L, x_{1}\right) \\
P_{m}\left(L, x_{2}\right) \\
\vdots \\
P_{m}\left(L, x_{M}\right)
\end{array}\right\},[G]=\left[\begin{array}{cccc}
\frac{K_{m 1}}{k} & 0 & \ldots & 0 \\
0 & \frac{K_{m 2}}{k} & \cdots & 0 \\
\vdots & \vdots & \ddots & \vdots \\
0 & 0 & \cdots & \frac{K_{m M}}{k}
\end{array}\right]
\end{gathered}
$$

The solution to Eqs. (29) and (30) is

$$
\begin{gathered}
-i k\{P\}=[Z]\{U\} \\
{[Z]=[C][G]^{-1}[C]^{-1}}
\end{gathered}
$$

Equation (34) is a nonlocal, nonreflecting boundary condition that allows all waves to exit the exit plane without reflecting. This boundary condition has the disadvantage that the matrix $[Z]$ is a dense matrix that must be numerically computed. On the other hand, this most general form of the boundary condition contains each and every propagating mode, is not dependent on the sound source, and is valid in flows with or without mean shear. Note that when a single mode, $(m n)$, exists at the termination, the boundary condition reduces to the well-known, local boundary condition for a single outgoing wave in a duct

$$
\{U\}=-i K_{m n}\{P\}
$$

The dimensionless node impedance matrix, [Z], can be fully determined once the mode shape $Q_{m n}(x)$ in the vertical direction and axial wave numbers $K_{m n}$ are determined. We now derive expressions for these quantities assuming that the upper and lower walls of the duct downstream of the trailing edge of the test liner are lined with uniform acoustic material. The analysis will assume uniform flow for the sake of simplicity, but it is easily extendible to the case of a parallel shearing mean flow. In the case of uniform mean flow, the eigenmode in the vertical direction $\left(Q_{m n}(x)\right)$ is obtainable using separation of variables

$$
Q_{m n}(x)=\left\{\begin{array}{ll}
\cos \left(\lambda_{n} x\right)+\frac{i k \alpha_{m n}}{\lambda_{n} \zeta_{l}} \sin \left(\lambda_{n} x\right), & \text { for } \lambda_{n} \neq 0 \\
1, & \text { for } \lambda_{n}=0, \text { or } \alpha_{m n}=0
\end{array}\right\}
$$

where $\lambda_{n}$ are complex zeroes of the transcendental function

$$
\begin{gathered}
E\left(k, H, M_{0}, \zeta_{l}, \zeta_{u}, \lambda_{n}\right)=i k \alpha_{m n} \lambda_{n}\left(\frac{1}{\xi_{l}}+\frac{1}{\xi_{u}}\right)-\left(\frac{k^{2}}{\xi_{l} \zeta_{u}} \alpha_{m n}^{2}+\lambda_{n}^{2}\right) \tan \left(\lambda_{n} H\right) \\
\alpha_{m n}=1-2\left(\frac{M_{0} K_{m n}}{k}\right)+\left(\frac{M_{0} K_{m n}}{k}\right)^{2} \\
7 \text { of } 15
\end{gathered}
$$




$$
\frac{K_{m n}}{k}=\frac{M_{0} \pm \sqrt{1-\left(1-M_{0}^{2}\right)\left[(m \pi / k W)^{2}+\left(\lambda_{n} / k\right)^{2}\right]}}{-\left(1-M_{0}^{2}\right)}
$$

Note that for a given value of $\lambda_{n}$, the axial propagation constant $K_{m n}$, satisfying Eq. (40), has two roots. Here, we will choose the root that corresponds to right moving waves in the duct.

The method of analysis described above requires a calculation of the mode eigenvalues, $\lambda_{n}$, that are the complex zeroes of the transcendental function, $E\left(k, H, M_{0}, \zeta_{l}, \zeta_{u}, \lambda_{n}\right)$. The method used to obtain these roots parallels the analytic continuation method discussed in Ref. 10 and is not discussed in further detail here. It should be noted that in most flow impedance tubes, the section of duct downstream of the trailing edge of the test liner contains rigid walls (i.e., $\left.\zeta_{u}=\zeta_{l}=\infty\right)$ so that the zeroes for the transcendental function, $E\left(k, H, M_{0}, \infty, \infty, \lambda_{n}\right)$, are known

$$
\lambda_{n}=\frac{n \pi}{H}, n=0,1,2, \ldots \infty
$$

and the axial propagation constants can be determined exactly

$$
\frac{K_{m n}}{k}=\frac{M_{0} \pm \sqrt{1-\left(1-M_{0}^{2}\right)\left[(m \pi / k W)^{2}+(n \pi / k H)^{2}\right]}}{-\left(1-M_{0}^{2}\right)}
$$

\section{Impedance Eduction Technique}

$\mathrm{T}$ HE numerical method chosen to solve Eqs. (20)-(22) for uniform flow and Eqs. (23)-(26) for parallel shearing flows, along with the nonreflecting boundary condition, closely parallels that used in an earlier paper ${ }^{3}$ for the zero-order spanwise mode (i.e., $m=0$ ). It is assumed that there are $N$ and $M$ evenly spaced nodes in the axial and vertical directions of the duct, respectively. A finite element method with cubic Hermite polynomial basis functions and a rectangular element ${ }^{11}$ is applied to obtain the solution for $P_{m}(z, x)$. Integrating the second derivative terms in the partial differential equation by parts allows the wall impedance and nonreflecting boundary condition to be incorporated into the methodology using a weak formulation. The finite element method results in a discrete system of equations of the form

$$
[S]\{\Phi\}=\{B\}
$$

where $[S]$ is the sparse, complex, asymmetric stiffness matrix with order $4 M N$. We use an asymmetrical, parallel, direct sparse solver to obtain $\{\Phi\}$. The solver employs a compressed column storage scheme to reduce storage overhead. Only the nonzero coefficients within $[S]$ are stored, along with two pointer arrays that store the column numbers and starting indices of these nonzero coefficients. To achieve efficient solutions, the sparse solver uses two accelerators: equation reordering ${ }^{12,13}$ to reduce fill during the factorization of $[S]$ and parallelization. (The equation solver runs on multiple processors simultaneously.) Stewart's adaptation of the Davidon-Fletcher-Powell (SDFP) optimization algorithm $^{14}$ is used to determine the unknown impedance, $\zeta$, by minimizing the objective function

$$
F(\zeta)=\sum_{J=1}^{N}\left[P_{m}^{\mathrm{FEM}}\left(z_{J}, H\right)-P_{m}^{\mathrm{Meas}}\left(z_{J}, H\right)\right]\left[P_{m}^{\mathrm{FEM}}\left(z_{J}, H\right)-P_{m}^{\mathrm{Meas}}\left(z_{J}, H\right)\right]^{*}
$$

\section{Results and Discussion}

N in-house computer code (FEMQ3D) that implements the impedance eduction methodology discussed in this Apaper has been developed. The code uses F90 Fortran with double precision (i.e., 64 bit) arithmetic and was designed to run in a multiprocessor environment using a shared memory implementation. This approach has been chosen because the primary hardware to be utilized was a Silicon Graphics Origin 3000 system. In the current implementation the resistance, $\theta$, and reactance, $\xi$, of the test liner are the design variables $(\xi=\theta+i \xi)$. The optimizer (i.e., SDFP) runs only in sequential mode using central finite differences to compute the gradient of the objective function. The finite difference step size is $1 \times 10^{-8}$, and a stopping criteria of $1 \times 10^{-8}\left(F(\zeta) \leq 1 \times 10^{-8}\right)$ is used to terminate the search. Finally, we have accelerated the impedance eduction methodology by running the equation solver in parallel on eight central processing units (CPUs).

Two examples are provided to demonstrate the accuracy, efficiency, and robustness of the impedance eduction methodology presented in this paper. In the first example we demonstrate the ability of the methodology to converge 
to the known impedance of a ceramic tubular (CT57) uniform test liner. This example contains two design variables, the resistance and reactance of the uniform liner. In the second example, we show that the method can educe the known impedances of a four-segment liner, in which three segments contain conventional liner configurations (three different perforated facesheet-over-honeycomb core combinations) and one segment is a CT57 liner. This second example contains eight design variables, the resistance and reactance of each of the four liner segments.

We desire to implement this quasi-3-D methodology for the LSFD currently being brought on-line at NASA LaRC. Since this duct is not yet fully operational, measured data are not available. Therefore, for the current study, we use synthesized data based on impedances educed from data acquired in the GIT. For the first example, the impedance of the CT57 liner is used as input to the exact modal solution for outgoing waves in an infinite duct (see the discussion at the end of section VI). To simulate the infinite duct, we set $L_{1}=0.0$ and $L_{2}=L$ (see Fig. 1). It should be noted that for each spanwise mode order $(m)$ and frequency $(f)$, the mode with the lowest decay rate is used as the sound source for this example.

Unfortunately, exact solutions are not available to synthesize acoustic pressure data for a four-segment liner in the LSFD geometry. Instead, a finite element code was used to synthesize data for the four-segment liner by incorporating the known impedances of each of the four segments. For this study, the first, second and fourth segments were conventional liner segments, and the third segment was a CT57 liner segment. Each segment is four inches long and eight-inch sections of rigid wall are located upstream and downstream of the leading and trailing edge of the four-segment liner. We used a plane wave as the sound source in the hard wall duct for this example.

Both examples (i.e., the uniform CT57 liner and four-segment liner) use a nonlocal, nonreflecting boundary condition (Eq. (34)) to terminate the duct. Also, the SDFP optimization is initialized using normalized resistance and reactance values of 1.0 and -1.0 , respectively. Further, a uniform spatial grid with 65 points $(N=65)$ in the axial and 17 points $(M=17)$ in the vertical directions, respectively, was used to educe the impedances. Thus, the node impedance matrix, $[Z]$, used in the computation was a $17 \times 17$ dense matrix. It should be noted that since there are slightly more than seven wavelengths in the axial direction (for a hard-wall duct), the chosen spatial grid is approximately three times finer than is needed in that direction.

The impedances for each example are educed under conditions for which the liner is located on the upper wall and higher-order spanwise modes are cut on. These conditions are implemented for a range of frequencies and flow Mach numbers. The frequency ranges from 0.5 to $3.0 \mathrm{kHz}$ (in increments of $0.1 \mathrm{kHz}$ ) and the centerline Mach numbers are $0.0,0.2$, and 0.4. To limit the scope of our investigation, we present results only for uniform mean flow. It is our intention to present results with shear at a later time.

Before proceeding to the impedance eduction studies, it should be noted that a typical impedance eduction run (i.e., for a single Mach number, frequency, and spanwise mode number) using FEMQ3D on a single CPU requires slightly less than 40 seconds of wall clock time and 200 megabytes of RAM on our Silicon Graphics ORIGIN 3000 computer platform. Additionally, the new sparse solver is nearly an order of magnitude faster than the now antiquated band solver used in our earlier papers. ${ }^{2-4}$ Additionally, results presented in the current paper were achieved by running the sparse equation solver in parallel on 8 CPUs. This reduced the wall clock time from 40 seconds (on a single $\mathrm{CPU}$ ) to slightly less than six seconds (using $8 \mathrm{CPUs}$ ) for each optimization run. These large reductions in wall clock turnaround times should allow the current method to be applied to a fully 3-D problem.

\section{A. CT57 Liner}

$\mathrm{T}$

HE resistance and reactance educed in the CT57 liner (from synthesized data) in the LSFD at zero Mach number $\left(M_{0}=0.0\right)$ are plotted in Fig. 2 . We use a dual-axis system with the resistance and reactance plotted on the y1 and y2 axis, respectively. Results obtained for each spanwise mode, $m$, in the LSFD are plotted against those educed in the GIT. To the level of resolution presented in Fig 2, the impedance educed in the LSFD is the same for each spanwise mode and reproduces exactly that educed (from measured data) in the GIT. For this reason, we have plotted the GIT and the LSFD results for each spanwise mode order as a single curve in Fig. 2. This excellent agreement is evidenced by the coalescence of four curves (one for the GIT and three for the individual spanwise modes in the LSFD) for each component of impedance, and results from the fact that an extremely fine finite element spatial grid was used. It is also clear that the small values of the finite difference step sizes and stopping criterion used in SDFP are also important parameters contributing to such excellent agreement. The number of iterations that SDFP requires to educe the impedance is an increasing function of the spanwise mode order. As an example, the second order spanwise mode $(m=2)$ typically requires four times the wall clock time as that of the zero order spanwise mode $(m=0)$.

Results are given in Figs. 3 and 4 for mean flow average Mach numbers (averaged across the duct cross-section) of 0.172 and 0.335 , respectively. Recall that the impedance of the CT57 liner was obtained in the GIT where boundary layers were present. Note, this uniform flow calculation ignores boundary layer effects. These uniform Mach 
numbers were used with the LSFD synthesized data to educe the impedance. The key feature of this exercise was to demonstrate self-consistency of FEMQ3D when higher-order modes are included. It is also an indication that the nonlocal, nonreflecting boundary condition (Eq. (34)) is functioning properly in the presence of grazing flow. Again, it was observed that the SDFP required many more iterations (and thus, significantly more wall clock time) to educe the impedance when higher-order spanwise modes were propagating in the duct.

A major drawback introduced by the use of local, gradient-based optimizers (such as SDFP) is that they often converge to a local minimum. In this situation, different design points (i.e., impedances) may be obtained if the optimizer is initialized from different points in the design space. The reduced wall clock turnaround time of FEMQ3D has allowed the authors to perform exhaustive studies of the effects of the starting location. The SDFP was initialized at 100 distinct starting locations and was found to converge to the same value of impedance for 95 of these starting locations. The SDFP did not converge for the remaining five starting locations because of a tendency of the gradient search technique to stall on flat surfaces. This clearly indicates that the objective function (Eq. (44)) contains a single minimum point for the uniform liner.

\section{B. Four-Segment Liner}

$\mathrm{T}_{\mathrm{i}}^{\mathrm{s}}$ HE second example presented is for the four-segment liner. The current quasi-3-D methodology allows the impedance of each of the four segments to be educed in the presence of flow. This is a significant enhancement over the previous impedance eduction methodologies implemented by the authors. Educed impedances were obtained by initializing each of the four impedance segments with $\zeta=1.0-1.0 i$ in the SDFP optimization. Because the impedances of the three conventional liner segments were only known for average mean flow Mach numbers of 0.172 and 0.335 , and for frequencies of $0.5,1.0,1.5,2.0,2.5$, and $3.0 \mathrm{kHz}$, computed results are restricted to these ranges of parameters.

Figures 5a, 5b, 5c, and 5d show the educed resistance and reactance values for the first, second, third, and fourth segments, respectively. The average mean flow Mach number is 0.172 , and results are plotted only for the zero order spanwise mode $(m=0)$ and first-order $(m=1)$ spanwise modes. With the exception of the anti-resonance frequency $(2.0 \mathrm{kHz})$ results for the third segment (CT57), the educed resistance and reactance of each segment is nearly the same (as expected) for each spanwise mode ( $m=0$ and $m=1)$. Also, these values are well matched to those educed in the GIT. The discrepancy in the impedance of the third segment (see Fig. 5c) at the antiresonance frequency has been observed in the 2-D analysis in an earlier paper $^{3}$ and is believed to be due to the sensitivity of the sound field to small changes in impedance near the antiresonant frequency. Segmented liner impedance eductions for an average mean flow Mach number of 0.335 are shown in Figs. 6a-6d. Trends at this higher Mach number are consistent with those in Figs. 5a-5d.

One complication in the four-segment liner impedance eduction is the increase in the number of SDFP stall points when compared to that of the uniform CT57 liner. This result is not that surprising, given that the function space has increased from a two-dimensional to an eight-dimensional design space. We have also experimented with different starting locations in SDFP for the four-segment liner. Each segment of the four-segment liner was initialized from the following six starting locations, $1+i, 1-i, 5+5 i, 5-5 i, 10+10 i$, and $10-10 i$. We observed that the SDFP converged to the same value of impedance as plotted in Figs. 5 and 6, except for the instances where the SDFP stalled on a flat surface and had to be restarted elsewhere in the design space.

\section{Conclusions}

$\mathrm{T}$ LE LaRC impedance eduction methodology has been extended to account for quasi-3-D sound fields in a uniform or shearing flow. A key element of the shear flow methodology is the introduction of a third order pressure equation that does not require a measurement of the inflow acoustic particle velocity for its implementation. A second important element of the current theory is the formulation of a nonlocal, nonreflecting boundary condition to terminate the duct when higher-order spanwise modes are cut on. The introduction of a parallel, sparse, equation solver has significantly reduced the wall clock time for educing the impedance when compared to that of the band solver used in earlier implementations. Results presented here have shown that the uniform flow methodology consistently reproduces the same impedance as that achieved for the zero order spanwise mode when several higher order spanwise modes ( $m=1,2)$ are cut on, thus demonstrating inter-code consistency. This work also illustrates that the uniform flow methodology is capable of educing the impedance of a multisegmented liner. This ability should significantly reduce the amount of time and cost required to determine the impedance of several uniform liners by allowing them to be placed in series in the test section, and the impedance of each segment educed using a single experiment. Finally, we 
have demonstrated the robustness of using the known acoustic pressure on a wall opposite the test liner to construct the objective function for the impedance eduction. This objective function contains only a global minimum and is, therefore, ideal for use with local, inexpensive, gradient-based optimizers. Also, the sheared flow impedance eduction methodology needs to be tested and validated.

\section{References}

1 Armstrong, D. L., “Acoustic Grazing Flow Impedance Using Waveguide Principles,” NASA CR-120848, 1971.

${ }^{2}$ Watson, W. R., Jones, M. G., Tanner, S. E., and Parrott, T. L., “ A Finite Element Propagation Model for Extracting Normal Incidence Impedance in Nonprogressive Acoustic Wave Fields," Journal of Computational Physics, Vol. 125, No. 1, April 1996, pp.177-186.

${ }^{3}$ Watson, W. R., Jones, M. G., and Parrott, T. L., "Validation of an Impedance Eduction Method in Flow," AIAA Journal, Vol. 37, No. 7, July 1999, pp. 818-824.

${ }^{4}$ Watson, W. R., Tracy, M. B., Jones, M. G., and Parrott, T. L., "Impedance Eduction in Shear Flow," AIAA Paper 2001-2263, Proceedings of the 7th AIAA/CEAS Aeroacoustics Conference \& Exhibit, Maastricht, The Netherlands, May 28-30, 2001.

5 Jones, M. G., Parrott, T. L., and Watson, W. R., "Comparison of Acoustic Impedance Eduction Techniques for Locally-Reacting Liners," AIAA Paper 2002-2246, Proceedings of the 8th AIAA/CEAS Aeroacoustics Conference \& Exhibit, Breckenridge, CO., June 17-19, 2002.

6 Jones, M. G., Watson, W. R., and Parrott, T. L., "Design and Evaluation of Modifications to the NASA Langley Flow Impedance Tube," AIAA Paper 2004-2837, Proceedings of the 10th AIAA/CEAS Aeroacoustics Conference \& Exhibit, Manchester, United Kingdom, May 12-14, 2004.

${ }^{7}$ Kraft, R. E., "Theory and Measurements of Acoustic Wave Propagation In Multi-Segmented Rectangular Ducts," P.h.D. Thesis, University of Cincinnati, Cincinnati, Ohio, 1976.

${ }^{8}$ Myers, M. K., "On The Acoustic Boundary Condition In The Presence Of Flow," Journal Of Sound And Vibrations, Vol. 71, No. 3, 1980, pp. 429-434.

9 Zorumski, W. E., Watson, W. R., and Hodge, S. L., "A Nonlocal Computational Boundary Condition for Duct Acoustics," Journal of Computational Acoustics, Vol. 3, No.1, June 1995, pp. 15-26.

10 Snider, Royce, Attenuation Predictions for Segmented Liners in Supersonic Flow. MS Thesis, The George Washington University, Washington, D. C., July 1993.

${ }^{11}$ Desai, C., and Abel, John F., Introduction To The Finite Element Method: a Numerical Method for Engineering Analysis, Van Nostrand Reinhold Company, New York, NY, 1972.

${ }^{12}$ Liu, J., "Modification of the Minimum-Degree Algorithm by Multiple Elimination," Association for Computing Machinery, Transactions on Mathematical Software, Vol. 11, No. 2, June 1985, pp. 141-153.

13 George, A., and Liu, J., Computer Solution of Large Sparse Positive Definite Systems, Prentice-Hall, Inc., Englewood Cliffs, NJ, 1981, Chaps. 5, 10.

14 Stewart, C. W. III, “A Modification of Davidon's Minimization Method to Accept Difference Approximations of Derivatives," Journal of ACM, Vol. 14, No. 1, Jan. 1967, pp. 72-83. 


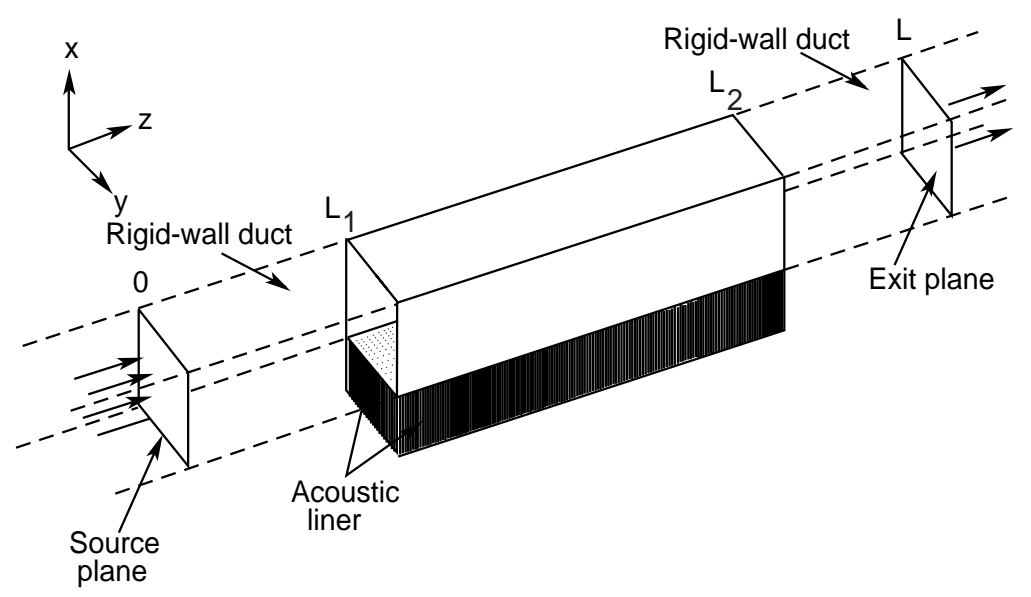

Figure 1. Schematic of 3-D duct, computational domain, and acoustic test liner.

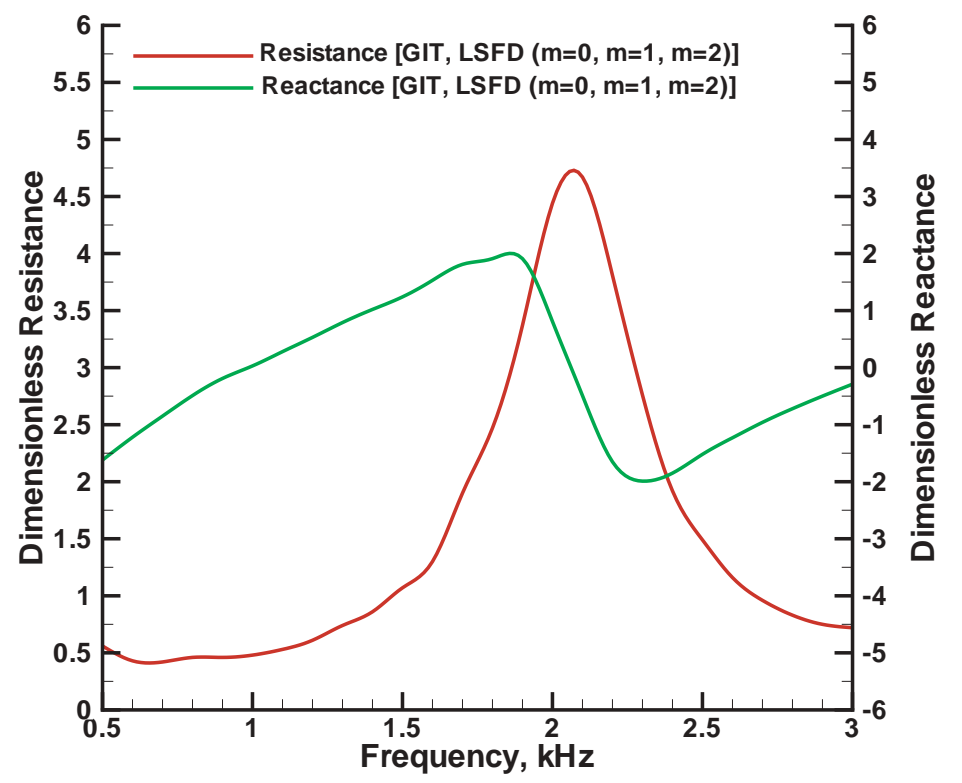

Figure 2. Educed impedance for CT57 liner in LSFD with synthesized data compared to measured data in GIT $\left(M_{0}=0.0\right)$. 


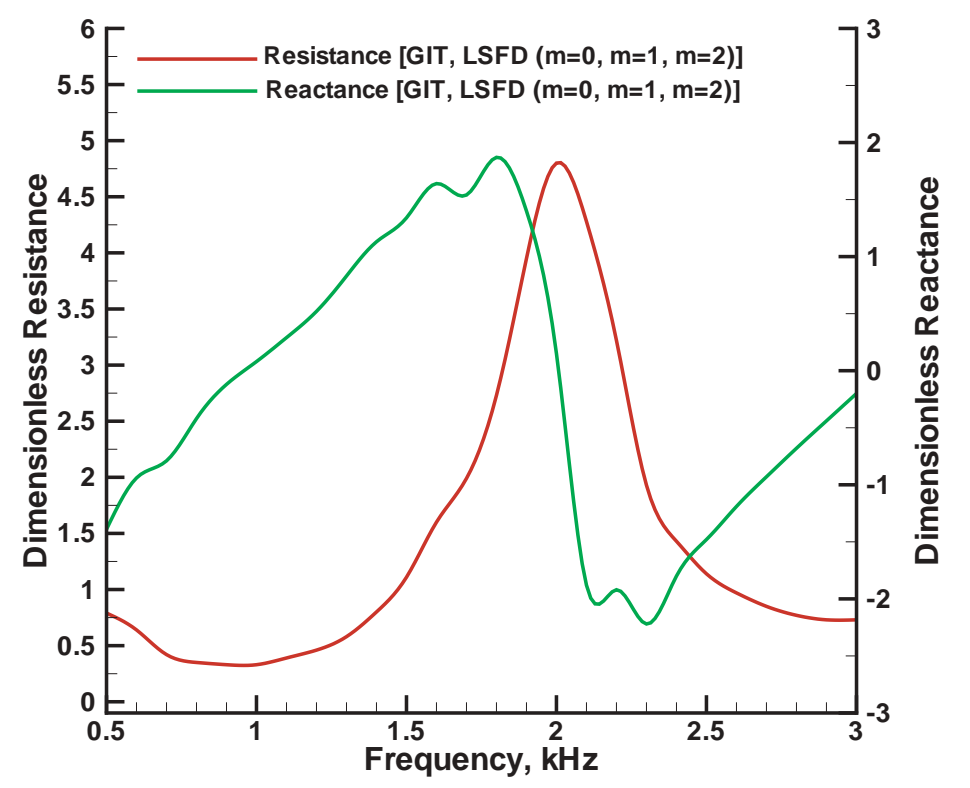

Figure 3. Educed impedance for CT57 liner in LSFD with synthesized data compared to measured data in GIT $\left(M_{0}=0.172\right)$.

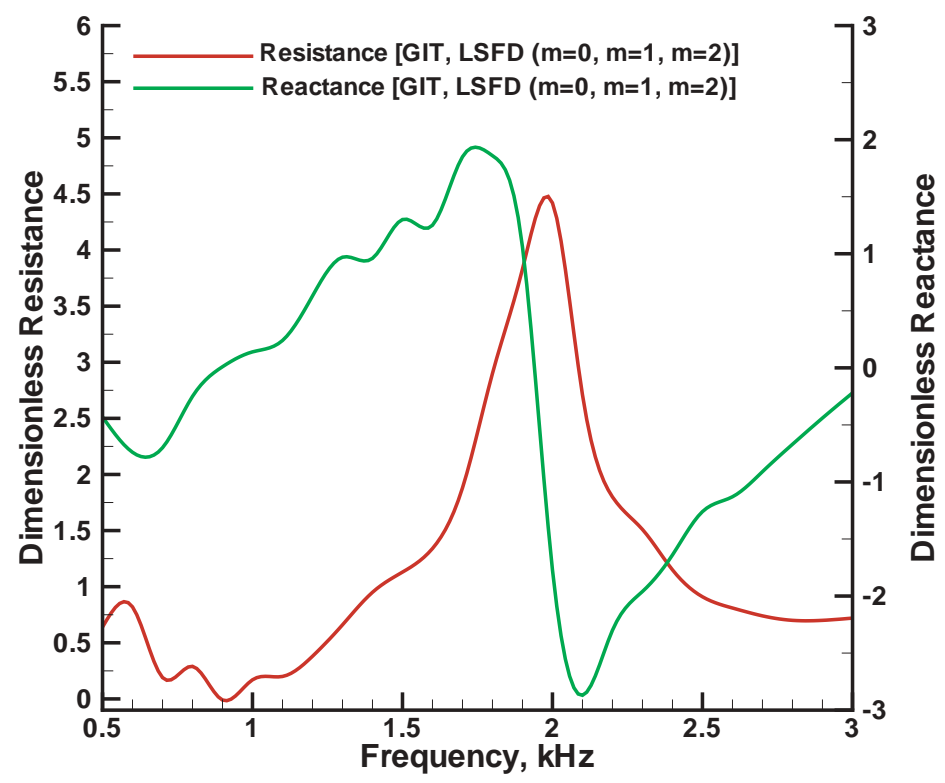

Figure 4. Educed impedance for CT57 liner in LSFD with synthesized data compared to measured data in the GIT $\left(M_{0}=0.335\right)$. 


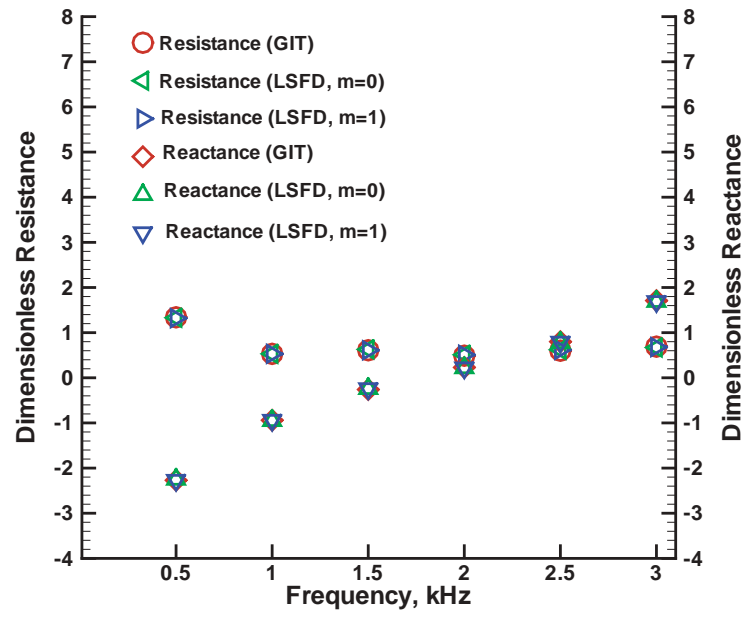

(a) Impedance of first segment

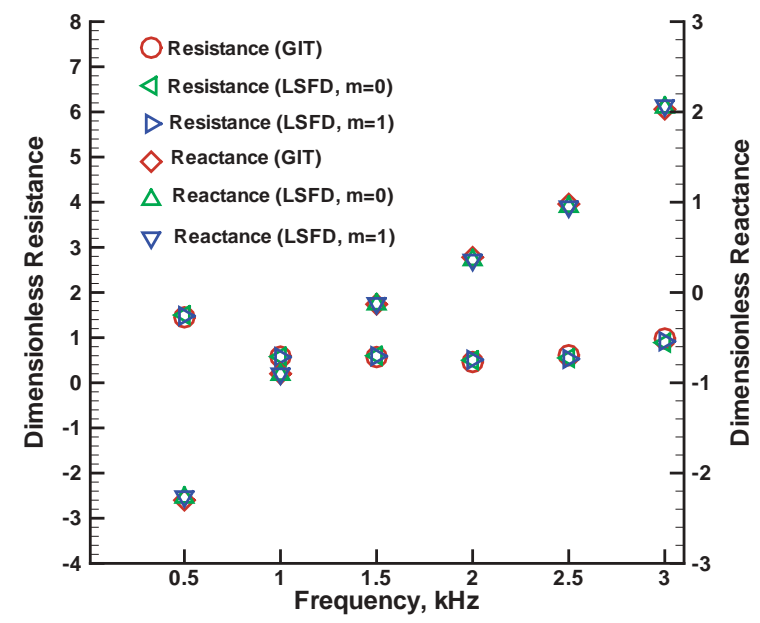

(b) Impedance of second segment

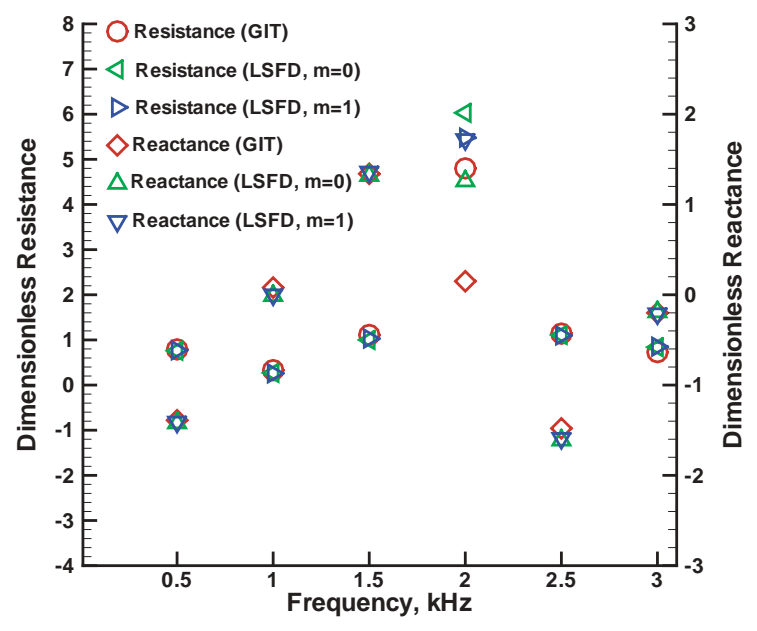

(c) Impedance of third segment

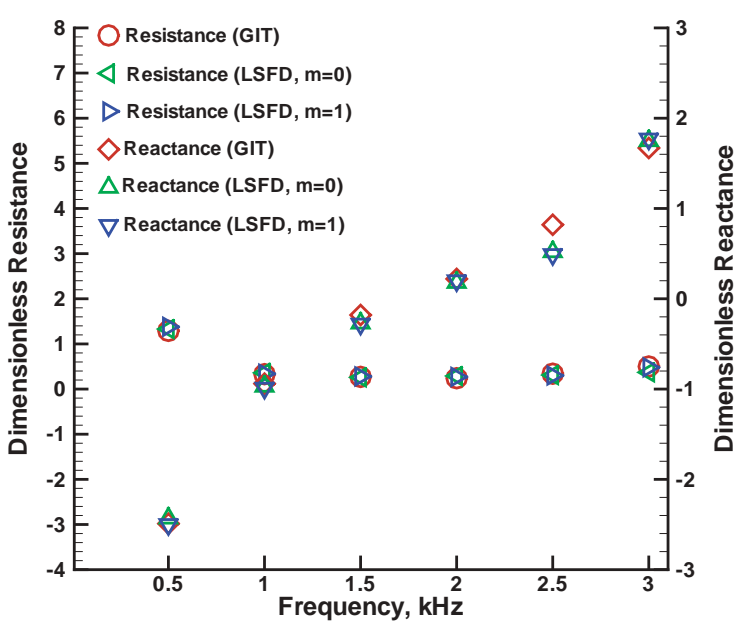

(d) Impedance of fourth segment

Figure 5. Educed impedance of four-segment liner in LSFD with synthesized data compared to measured data in GIT at Mach 0.172. 


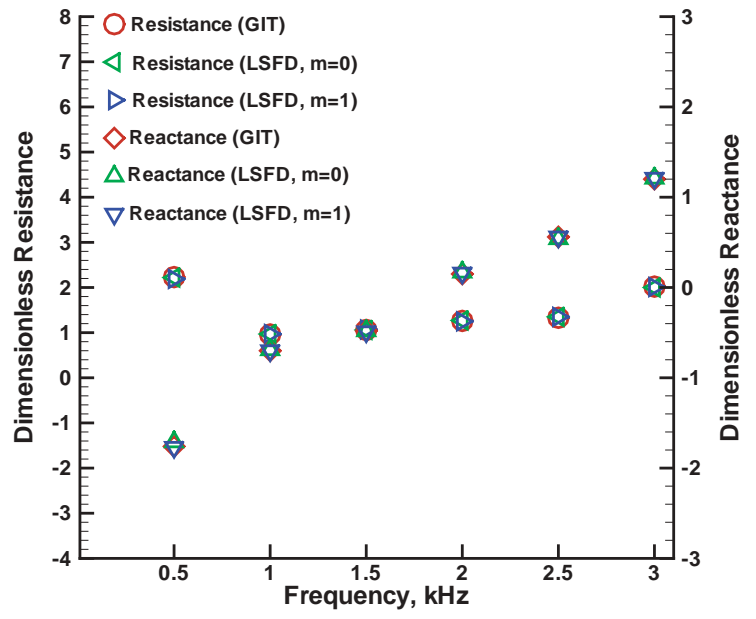

(a) Impedance of first segment

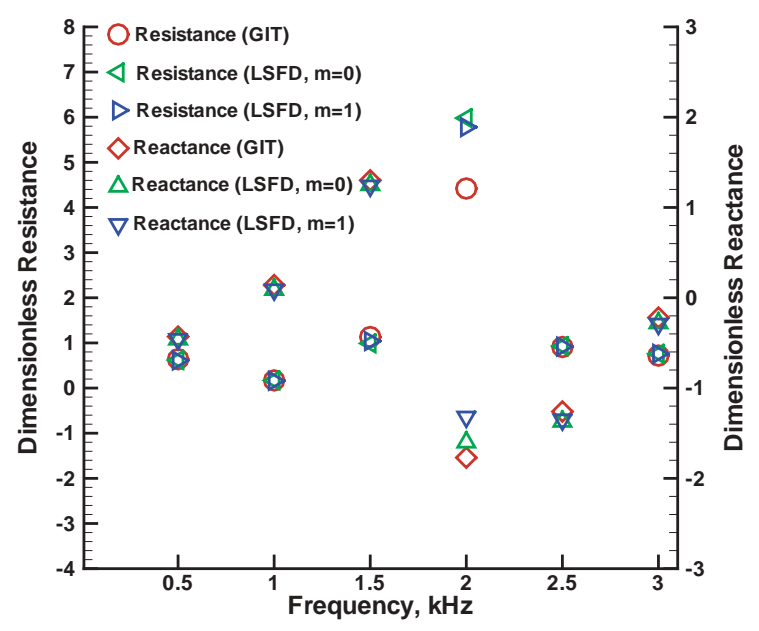

(c) Impedance of third segment

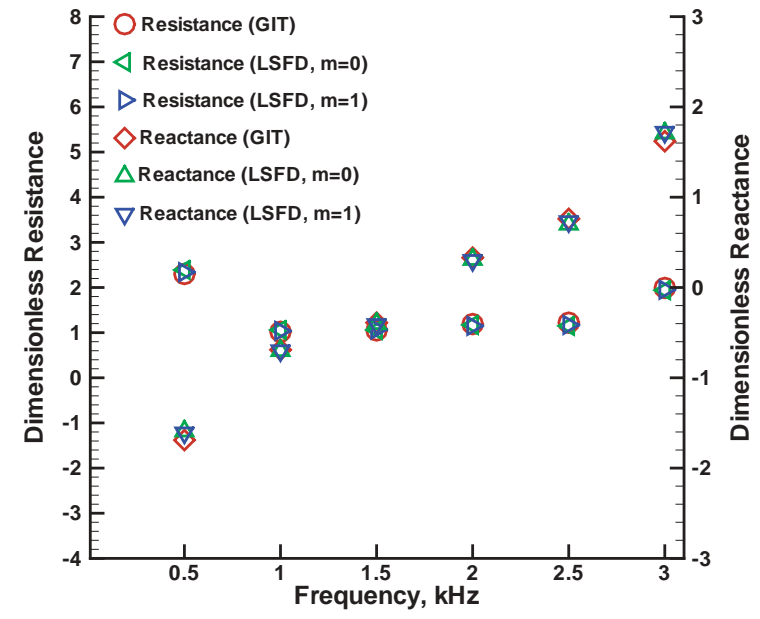

(b) Impedance of second segment

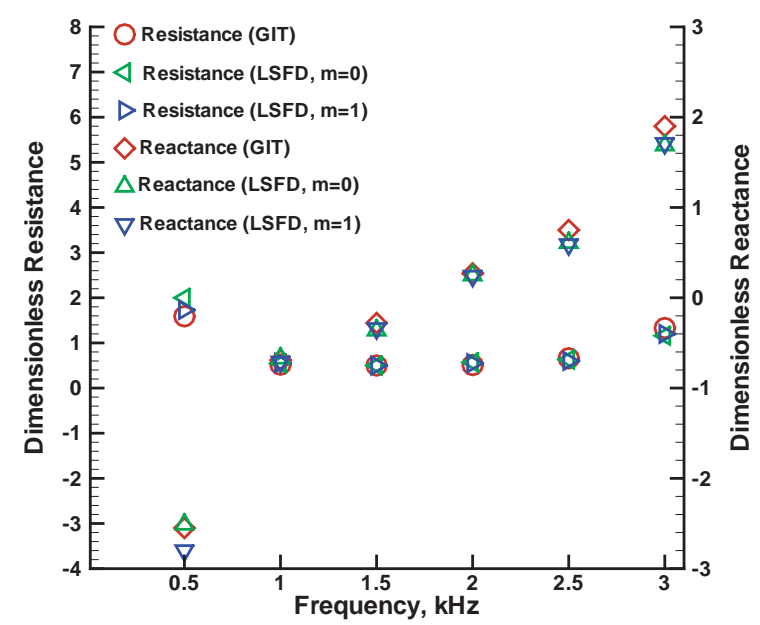

(d) Impedance of fourth segment

Figure 6. Educed impedance of four-segment liner in LSFD with synthesized data compared to measured data in GIT at Mach 0.335. 